\title{
The Iron Abundance in Blue Compact Galaxies
}

\author{
Rodríguez, M. \\ Instituto Nacional de Astrofísica, Óptica y Electrónica, INAOE, Apdo. \\ Postal 51 \& 216, Puebla, Pue 72000, Mexico \\ Esteban, C. \\ Instituto de Astrofísica de Canarias, IAC, E-38200 La Laguna, \\ Tenerife, Spain
}

\begin{abstract}
We determine the Fe abundances for several blue compact and emission-line galaxies. The $\mathrm{O} / \mathrm{Fe}$ abundance ratios are found to increase with the metallicity of the galaxy, and to decrease with its degree of ionization. These correlations are interpreted as reflecting a decrease for lower metallicities of the depletion of Fe atoms on to dust grains, probably arising from the harder radiation fields prevailing at low metallicities.
\end{abstract}

\section{Results and discussion}

We present the first results of an analysis of the Fe abundance in blue compact and emission-line galaxies. This analysis improves on previous ones (see Izotov \& Thuan 1999) since it is based on (1) the measurement of at least two [Fe III] lines, (2) the atomic data for $\mathrm{Fe}^{++}$that best reproduce the relative intensities of the [Fe III] lines (see Rodríguez 2002), and (3) new calculations of ionization correction factors (ICFs) from photoionization models that incorporate recently improved values for the relevant atomic data. Our new results for the $\mathrm{O} / \mathrm{Fe}$ abundance ratio differ from the previous ones by factors up to 2 . Since the $\mathrm{O} / \mathrm{Fe}$ abundance ratio provides important constraints on the history of star formation in a galaxy and on the processes of stellar nucleosynthesis (e.g. Recchi et al. 2002), these new results are of relevance to these issues.

We obtained optical CCD spectra with the ISIS spectrograph on the 4.2 m WHT (Observatorio del Roque de los Muchachos, La Palma, Spain). We measured at least two [Fe III] lines for each object ( $\lambda 4658$ and the blend $\lambda 4985+$ 6 ), along with the lines needed to derive the $\mathrm{O}$ abundance, the extinction and the physical conditions. Only [Fe III] $\lambda 4658$ had been measured previously in most of these galaxies; the measurement of two [Fe III] lines allows us to rule out contamination with C IV $\lambda 4658$ in those galaxies with Wolf-Rayet features. Ionic abundances were obtained with the nebular package in IRAF, except for $\mathrm{Fe}^{++}$(see Rodríguez 2003). The $\mathrm{O} / \mathrm{Fe}$ abundance ratio was derived from the relation (Rodríguez \& Rubin, this volume): $\mathrm{Fe} / \mathrm{O}=\left(\mathrm{O}^{+} / \mathrm{O}^{++}\right)^{0.09}\left(\mathrm{Fe}^{++} / \mathrm{O}^{+}\right)$.

The resulting abundance ratios are presented as a function of $\mathrm{O} / \mathrm{H}$ in Figure $1 \mathrm{a}$, where $[\mathrm{O} / \mathrm{Fe}]=\log (\mathrm{O} / \mathrm{Fe})_{\text {galaxy }}-\log (\mathrm{O} / \mathrm{Fe})_{\odot}$, and $\log (\mathrm{O} / \mathrm{Fe})_{\odot}=1.29$ 

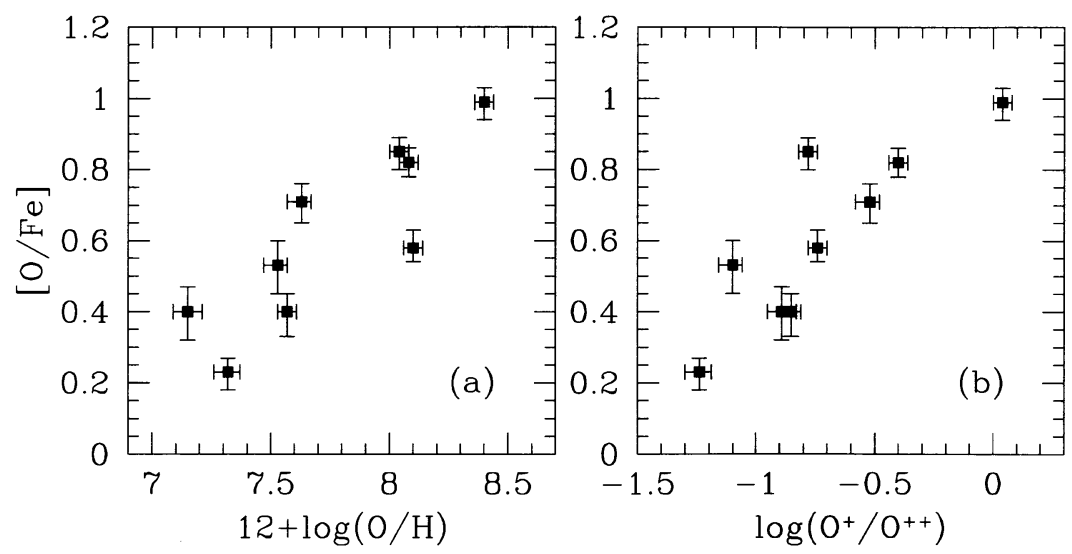

Figure 1. $[\mathrm{O} / \mathrm{Fe}]$ abundance ratios as a function of the $\mathrm{O}$ abundance (a) and the degree of ionization (b). In order of increasing $\mathrm{O}$ abundance, the results correspond to I $\mathrm{Zw}$ 18, SBS 0335-052, SBS $0940+544 \mathrm{~N}$, SBS $1159+545$, SBS $1415+437$, Mrk 22, Mrk 1409, SBS $0948+532$, and NGC 1741 . The error bars only consider the uncertainties in the intensity ratios used to derive the extinction correction, the temperature, and the ionic abundances.

(Holweger 2001). There is a clear correlation between $[\mathrm{O} / \mathrm{Fe}]$ and $\mathrm{O} / \mathrm{H}$, probably reflecting an increase in the depletion of $\mathrm{Fe}$ atoms on to dust grains at higher metallicities. Figure $1 \mathrm{~b}$ shows that the values of $[\mathrm{O} / \mathrm{Fe}]$ are also correlated with the degree of ionization $\left(\mathrm{O}^{+} / \mathrm{O}^{++}\right)$. This correlation follows and extends to higher degrees of ionization the correlation found in Rodríguez (2002) for Galactic H II regions in the solar neighborhood. This suggests that the correlation is due to the release of Fe atoms from dust grains by the action of energetic photons both for the Galactic H II regions and for our sample galaxies.

Caveat. If the current discrepancy between the $\mathrm{Fe}$ abundances derived from [Fe III] and [Fe IV] lines (Rubin et al. 1997; Rodríguez 2003) is not due to errors in the insufficiently tested atomic data for $\mathrm{Fe}^{3+}$ but to errors in the $\mathrm{Fe}^{++}$atomic data or in the ICFs, the derived $\mathrm{O} / \mathrm{Fe}$ ratios will be higher by factors $\sim 2-4$.

Acknowledgments. This work was partially supported by Mexican CONACyT project J37680-E.

\section{References}

Holweger, H. 2001, in AIP Conf. Proc., 598, Solar and Galactic Composition, ed. R.F. Wimmer-Schweingruber (New York: Springer-Verlag), 23

Izotov, Y.I., \& Thuan, T.X. 1999, ApJ, 511, 639

Recchi, S., Matteucci, F., D'Ercole, A., \& Tosi, M. 2002, A\&A, 384, 799

Rodríguez, M. 2002, A\&A, 389, 556

Rodríguez, M. 2003, ApJ, 590, 296

Rubin, R.H. et al. 1997, ApJ, 474, L131 